\title{
Enhancement in Realistic Fluid Re-simulation
}

\author{
Yahui Song, Hongyan Quan* and Yuwen Huang \\ East China Normal University, Institute of Software Engineering, Shanghai, China \\ *Corresponding author
}

\begin{abstract}
Re-simulating a 3D fluid result from video while retaining and rendering details is significant in practice, which still remains a difficult task in spite of rapid advancements in this field during the last two decades. Physically driven models can be easily extended to handle fluid, yet they are unable to preserve surface details like breaking waves without the expense of increasing particle densities. This paper proposes a hybrid particle Lattice Boltzmann Model for Shallow Waters (LBMSW) coupling method to simulate fluid with finer details real-time from video. To preserve re-simulation surface details, we couple the detail particles with the distribution function of the LBMSW model. To improve the time performance, we use only surface to re-simulate. Experiments show that the proposed approach can obtain a realistic re-simulation products from video example in several challenging scenarios and we provide qualitative evaluation to the method.
\end{abstract}

Keywords-re-simulation; realistic details; fluid; LBMSW; ACE enhancement; velocity

\section{INTRODUCTION}

Fluid simulation has been widely used in fields of computer games, film special effects, military simulation and natural disasters defense. Although fluid simulation have made great progress recently, a realistic fluid simulations result in real time still remains a challenge in the relevant research areas.

The realistic detail plays a significant role in fluid simulation. In the previous studies, people introduce various methods to perform the simulation details. Wang et al. [1] propose a hybrid particle-grid coupling method to simulate fluid with finer details, which enhances the details with SPH particles. Thürey et al. [2] apply a multi-scale velocity scheme to preserve the fluid detail. How to re-simulate fluid real-time while keeping the details is popular recently. James et al. [3] couple fluid simulation into flow tracking, and provide a global prior to increase the tracking accuracy and temporal coherence to ensure the realistic re-simulation. To improve practicability, Quan et al. [4] use calculated physical properties to recover physically driven model to customize a desired naturalistic fluid re-simulation result. This paper applies the recovered physically driven model to re-simulate the fluid from the video.

The more desired result is, the more accurate the substantial information should be. To re-simulate longer artistic result in low level hardware, it is necessary to calculate the substantial information including the surface geometry and physical velocity. The traditional optical flow methods [5-6] use intensity constraint to compute the velocity while $\mathrm{Li}$ et al. [7] employ the shallow water to constrain to get physical-based velocity. For the water velocity, Quan et al. [4] propose a novel scheme to recover the $2 \mathrm{D}$ velocity in multi-scale frame work and get more accurate velocity field. Based on the work [4], we introduce the shallow water equation (SWE) in each-scale to refine the velocity, meanwhile get more accurate surface geometry. As the assumption of SWE is same with the physically driven model LBMSW, the calculated velocity is more effective for LBMSW re-simulation.

\section{SYSTEM OVERVIEW}

Our system inputs a single video of fluid scene and outputs realistic fluid 3D re-simulation. Figure 1 shows the pipeline of our 3D realistic re-simulation algorithm. The detail is as follows: after acquiring geometry from SFS [8], the velocity is calculated from dynamic behavior constrained by SWE, particle density and distribution function in LBMSW model are computed under the constraint of the surface geometry and the velocity. The contrast of density is enhanced to make up its decaying in the auto-advection. In the re-simulation module, LBMSW driven model is coupled with detail particles to retain the realistic details of re-simulation. The hash map is established between the heights of particle and colors to acquire realistic fluid appearance.

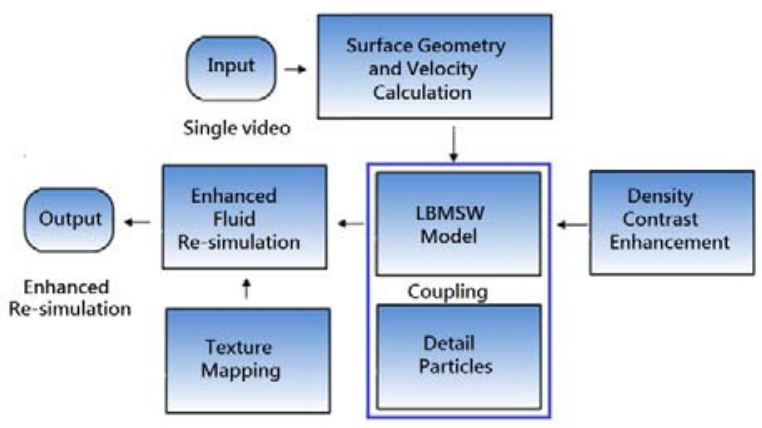

FIGURE I. THE PIPELINE OF OUR SYSTEM

\section{PhySiCALly-BASED REALISTIC RE-SIMULATION}

We apply adaptive density contrast enhancement algorithm to make up the density decaying in LBMSW model advection and introduce detail particles coupling with LBMSW to retain realistic details.

\section{A. Velocity Calculation in Multi-scale Framework}

The main idea of velocity calculation is that acquire the preliminary results from work [4] in each scale, and further refine the results using the constraint of physical principle 
which we introduce the SWE. The assumption of SWE is same with the physically driven model LBMSW, thus, the velocity constrained by SWE is effective for the re-simulation when render details automatically. Inspired by [5], we obtain the following energy function:

$$
E_{p}=\iint\left[\left(h_{t}+h_{x} u+h_{y} v+h\left(u_{x}+v_{y}\right)\right)^{2}+\beta^{2}\left(|\nabla u|^{2}+|\nabla v|^{2}\right)\right] d x d y .
$$

where $\beta$ is the Lagrange multiplier and is set 8.0 across all tests to control the combination weights. In this paper we use $\mathbf{u}(u, v)$ denote the 2D velocity along $(x, y)$ directions. $h(x, y)$ is height field of fluid surface particle from [4]. We set the time $t 1.0$ across all tests. The experiment demonstrates that the final result describes the complex water dynamics.

\section{B. Physically Driven Mode LBMSW}

Our fluid solver is LBMSW, in which the shallow water assumption is combined with D2Q9 based LBM model to deal with the 3D particle simulation. Under the constraint of shallow water model [11], the height value $h$ can be computed as: $\mathrm{h}=\rho$. Compared with other physical models, LBMSW can simulate in lower complexity and the boundary treatment can be easily realized. We take advantage of this model in this work. The Bhatnager-Gross-Krook model with singlerelaxation can be formulated as:

$$
f_{i}\left(\mathbf{x}+e_{i} \delta_{t}, t+\delta_{t}\right)=\left(1-\frac{1}{\tau}\right) f_{i}(\mathbf{x}, t)+\frac{1}{\tau} f_{i}^{e q} .
$$

where $e_{i}$ is the discrete particle velocity in the direction $i$, $f_{i}(\mathbf{x}, t), i=\{0,1,2 \cdots 8\}$ and $f_{i}^{e q}$ are the density distribution function and the local equilibrium density distribution function in the cell at $\mathrm{x}$ at time $t . \tau, \delta_{t}$ are the relaxation parameter and discrete time steps. We set the $\delta_{t} 1$ in all scene. The $f_{i}^{e q}$ [10] can be formulated as:

$$
f_{i}^{e q}(\rho, \mathbf{u})=\left\{\begin{array}{c}
\frac{4}{9}\left[1-\frac{1}{2 c^{2}} \mathbf{u}^{2}\right], i=0 \\
\lambda_{i} \rho\left[1+\frac{3}{c^{2}}\left(e_{i} \cdot \mathbf{u}\right)+\frac{9}{2 c^{4}}\left(e_{i} \cdot \mathbf{u}\right)^{2}-\frac{3}{2 c^{2}} \mathbf{u}^{2}\right]
\end{array} i=1,2 \cdots 8 .\right.
$$

In each discrete spatial cell, the density $\rho$ and velocity $\mathbf{u}$ can be computed as:

$$
\begin{aligned}
& \rho=\sum_{i=0}^{8} f_{i}(t) . \\
& \mathbf{u}=\frac{1}{\rho} \sum_{i=0}^{8} e_{i} f_{i} .
\end{aligned}
$$

\section{Density Enhancement}

We initialize the LBMSW model using first frame velocity and let it evolution automatically, we find that energy and particle densities of 3D fluid scene are redistributed and decayed. Then we propose an adaptive density contrast enhancement algorithm to preserve the details. We extract the high frequency of the density along four directions in spatial domain and enhance it linearly. Firstly, the density gradients of horizontal, vertical, direction of 45 degrees, 135 degrees are calculated separately, denoted as $\nabla \rho_{i}, i=\{0,45,90,135\}$. Then, they are smoothed with spatial mean filter and the results are denoted as $\nabla \rho_{i}^{s}, i=\{0,45,90,135\}$. Finally the high frequency component are enhanced linearly by:

$$
\nabla \rho_{i}^{e}=C_{e}\left(\nabla \rho_{i}-\nabla \rho_{i}^{s}\right), \quad i=\{0,45,90,135\}
$$

where $\nabla \rho_{i}^{e}$ denote the enhanced results of high frequency of density gradients of four directions respectively. Then the enhanced density $\rho_{e}$ can be enhanced from the auto-advection density $\rho_{a}$ by

$$
\rho_{e}=\left[1+\frac{1}{4} \sum_{k=1}^{4} \frac{\left(\nabla \rho_{d_{k}}^{e}+\nabla \rho_{d_{k}}^{s}\right)}{\nabla \rho_{d_{k}}}\right] \rho_{a}
$$

where $d_{k} \in\{0,45,90,135\}(\mathrm{k}=1 \cdots 4)$

After density enhancement, distribution functions are automatically enhanced in next advection period. In our study, we use the spatial mean smooth filter of $7 \times 7$ and set $C_{e} 2.8$ in the re-simulation, more satisfied results are obtained.

\section{Detail Particles}

To achieve re-simulation with rich details, we introduce detail particles to couple with LBMSW. Due to the detail particles are massless, this can keep mass conservative to whole 3D fluid scene. Certain features of geometry variation on fluid surface provide the cues to detect the details position. The detect position is necessary to satisfy the following two conditions [4] [12]:

$$
h_{t}-h_{t-1}>\Phi
$$

$$
\left\|\nabla h_{t}\right\|>\nabla h_{a}
$$

where $h_{t}, h_{t-1}$ are surface geometry of successive frames, $\Phi$ is a threshold parameter, which should be tailored per scene. $\nabla h_{t}$ denotes the spatial variation of geometry and is obtained from the difference between surface geometry $h_{t}$ and its local average $h_{s}: \nabla h_{t}=h_{t}-h_{s} \cdot \nabla h_{a}$ denotes the geometry gradient average of whole scene. We set variance $\sigma$ of Gauss filter 2 in $h_{s}$ calculation. 
The previous two conditions are necessary in detail positions detection. The former one is to detect the front in the wave with moving fast, and the later ensures that it is steep enough to generate the breaking or splash. The positions of detail particles are located within the cuboids centered by the detected position. The size of the cuboids can be calculated from particle interval, and the positions of the detail particles are disturbed from the detected position on grid. The number of the thrown particles $N$ at a certain detected position is calculated from the height difference $h_{p}$ :

$$
N=\frac{h_{p}}{r} .
$$

where $r$ is the particle radium obtained from half of particle interval in LBMSW. $h_{p}=h_{f}-h_{l}$, where $h_{f}$ is the surface geometry from SFS and $h_{l}$ is advection of LBMSW.

The velocities $\mathbf{v}$ of detail particles can be calculated from the advection height $h$ and advection velocity $\mathbf{u}$ in LBMSW.

$$
\mathbf{v}=\left[\sqrt{1+\frac{h_{p}}{h}}-1\right] \mathbf{u} .
$$

\section{E. Coupling between LBMSW and Detail Particles}

The detail particles and LBMSW model couple each other in re-simulation. The coupling framework is shown in Figure 2. The distribution functions $f_{i}$ of LBMSW are modified as

$$
f_{i}=f_{i}+f_{i}^{e q}\left(h_{p}, \mathbf{v}\right)
$$

where $f_{i}^{e q}\left(h_{p}, \mathbf{v}\right)$ denotes the momentum that the detail particles contained.

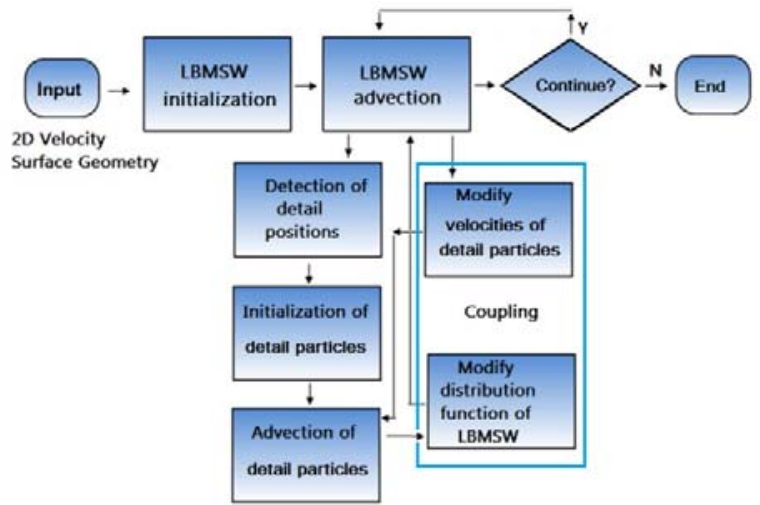

FIGURE II. COUPLING OF DETAIL PARTICLES AND LBMSW

\section{F. Texture Mapping}

To obtain realistic appearance consistency, hash maps between gray and color are established. Firstly, we normalize the particles height of the example frame to gray scale range $[0,255] . h_{x}, h_{m}$ stand for the maximum and minimum height. Then the gray value $g_{s}$ corresponding height value $h_{s}$ and gray value of particle $Y$ can be calculated as:

$$
g_{s}=\left\lfloor\frac{\left(h_{s}-h_{m}\right) \times 255}{h_{x}-h_{m}}\right\rfloor \text {. }
$$

$$
Y=0.3 R+0.59 G+0.11 B
$$

Here $R, G$ and $B$ are the three components of the color respectively. Thus the hash mapping $T$ from gray value to color can be established.

In order to map color to particle, gray value $g_{q}$ of the particle can be calculated by (14), and then color result can be obtained from hash map $T$ further. In the mapping procedure, seek failure and items conflict can be occurred. If gray value $g_{q}$ of the particle does not exist in $T$, and there exists height $g_{L}$ and $g_{H}$ in $T$ that satisfy $g_{L}<g_{q}<g_{H}$. If $\left|g_{L}-g_{q}\right|<\left|g_{H}-g_{q}\right|$, map the color related to $g_{L}$ to the particle, otherwise map the $g_{H}$. If there are several colors corresponding to gray value $g_{q}$ in $T$, select anyone of them to map the particle.

\section{RESUlts AND EVALUATION}

Results and quantitative evaluations of the realistic resimulation performance are provided here. All results are based on real captured data from Dyntex Dataset available to public [13]. We implement the algorithm in $\mathrm{C}++$, all fluid videos used in this study have unity resolution of 352 by 288 . Our hardware platform is PC with Intel (R) Pentium (R) 2.67GHz CPU, 4 GB memory. The re-simulation results are rendered with OpenGL library.

\section{A. Velocity Results and Performance}

We employ the SWE to constrain the calculation velocity. Figure 3 visually compares the velocity results from different methods. We use munsell color system to present the velocity. In each group, the lower is input video frame, the middle is the velocity result from [6] while the upper from our approach. From figure 3, we can see that the detail of the fluid is available from the last result.

To evaluate the velocity quality from different methods, errors of advection surface geometry are computed. The LBMSW model is initialized with the velocities from different methods separately, and then we calculate the average error as:

$$
E_{v}=\frac{1}{n} \sum_{i=1}^{n}\left|h_{i}-z_{i}\right|
$$

Here $n$ is the total number of particles, $h_{i}$ is advection height of particle $p$ and $z_{i}$ is surface geometry. 
The average errors of the 4th frame from different methods are listed in Table 1 . It can be easily seen that almost the statistic average errors from our method are relatively small, which shows that the velocities calculated from our method seem more satisfactory.

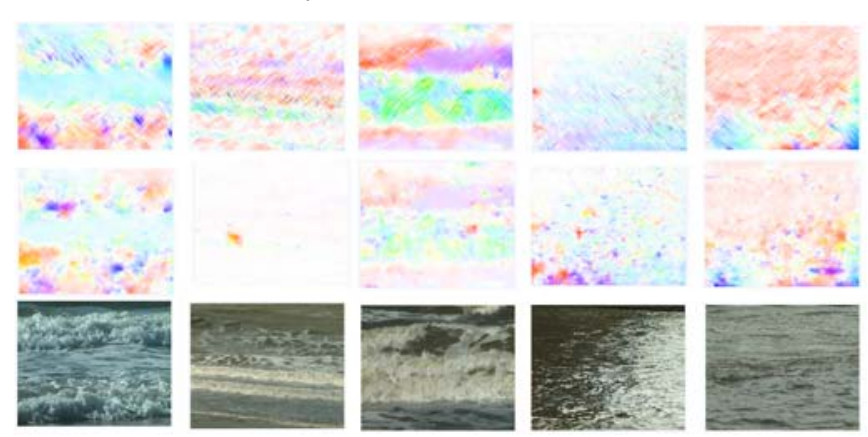

FIGURE III. THE VELOCITY COMPARISON RESULTS

TABLE I. AVERAGE ERROR STATISTICS RESUlTS

\begin{tabular}{|l|l|l|l|}
\hline \multirow{2}{*}{ Video } & \multicolumn{3}{|c|}{ Statistics Results $E_{v}$} \\
\cline { 2 - 4 } & \multicolumn{1}{|c|}{ Ref.[7] } & \multicolumn{1}{|c|}{ Ref.[5] } & Ours \\
\hline 54ab110 & 0.86148 & 0.868418 & 0.845329 \\
\hline 649ea10 & 0.865053 & 0.84753 & 0.766625 \\
\hline 649dd10 & 0.851859 & 0.766581 & 0.787117 \\
\hline 649dg10 & 0.881909 & 0.790548 & 0.715591 \\
\hline 649de10 & 0.875527 & 0.807761 & 0.765751 \\
\hline 649dc10 & 0.793682 & 0.729263 & 0.692097 \\
\hline 647b810 & 0.836226 & 0.843881 & 0.81406 \\
\hline
\end{tabular}

\section{B. Re-simulation Results}

Figure 4 shows the enhanced re-simulation results of different types of fluid, in each group the up one is the source frame from video, and bottom is the realistic enhanced resimulation result using our approach. It can be easily seen that the details can be retained in the hybrid particle LBMSW realistic re-simulation.
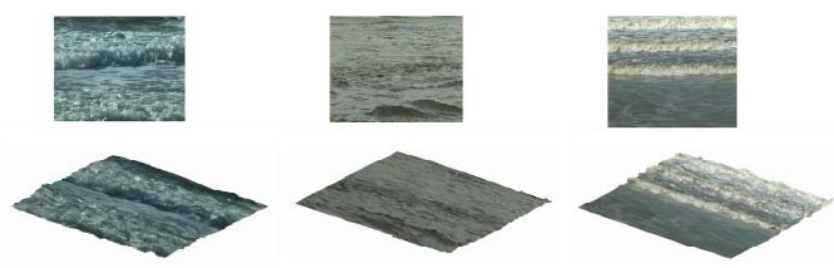

FIGURE IV. ENHANCED RE-SIMULATION RESULTS

\section{CONCLUSION}

We introduce a key technique for re-simulating 3D fluid real-time based on physical driven model. It combines constraints of appearance, dynamic behavior and physical principle of the example water to optimize velocity of the first frame. Further, density and distribution functions of the LBMSW can be acquired. In order to obtain a realistic derivative product, adaptive algorithm of density contrast enhancement makes up the density decaying in LBM advection. Particles and LBMSW are combined into a two-way coupling re-simulating system to retain realistic details. Our approach has the following advantages:

- Calculation of velocity which is constrained by the physical principle.

- An efficient density enhancement solution to the faded details, which makes sure the realistic re-simulation.

- A physically-based density enhancement to ensure the detail preservation.

There are limitations to overcome and it is necessary to extend study to improve this work. There are many applications for this approach. This study primarily focuses on re-simulation with complex fluid dynamic. How to learn about the variation of the physical properties through machine learning and further control the physical driven model is more important to be considered in the future work.

\section{ACKNOWLEDGMENT}

This work is also supported and funded by NSFC Grant No. 61070128, 61272199. And special thanks to the reviewers for their valuable comments and suggestions.

\section{REFERENCES}

[1] Wang Changbo, Zhang Qiang, Kong Fanlong, Qin Hong, "Hybrid particle-grid fluid anima-tion with enhanced details," The Visual Computer. Vol. 29, No. 9, pp. 937-947, 2013.

[2] N. Thürey, R. Keiser, M. Pauly, U. Rüde "Detail-preserving fluid control,” Graphical Mod-els. Vol. 71, No. 6, pp. 221-228, 2009.

[3] G.James, I.Ivo, T.Nils, H.Wolfgang, "From Capture to Simulation Connecting Forward and Inverse Problems in Fluids," ACM SIGGRAPH, 2014

[4] H. Quan, C. Wang, Y. Song, ”Fluid re-simulation based on physically driven model from video," The Visual Computer, 2015: 1-14.

[5] B. K. P. Horn and B. G. Schunck, ”Determing optical flow," Artificial Intelligence,vol. 17, pp. 185-203, 1981.

[6] M. Enric, S.Javier, K.Daniel,” Horn-Schunck Optical Flow with a Multi-Scale Strategy, "Image Processing On Line,vol. 3 , pp. 151-172, 2013.

[7] C. Li, D. Pickup, T. Saunders, D. Cosker, D. Marshall, P. Hall , P. Willis, "Water Surface Modeling from A Single Viewpoint Video," TVCG, pp. 1242-1251, 2013.

[8] M.Q.Yu, H.Y. Quan, "Fluid surface reconstruction based on specular reflection model," Computer Animation and Virtual Worlds, 24(5), 497510 (2013).

[9] C. Li, D. Pickup,T.Saunders, D.Cosker, D.Marshall, P. S. Hall, P. Willis, "Water surface modeling from a single viewpoint video," Visualization and Computer Graphics, IEEE Transactions on, 19(7), 1242-1251 (2013).

[10] P.Yuan, L. Schaefer, "Equations of state in a lattice Boltzmann model,” Physics of Fluids (1994-present), 18(4), 042101(2006).

[11] C.B.WANG, Z. ZHANG, Q. ZHANG, Y.QIAN, J. ZHANG, T. XU, ” Realistic Rendering of Free Surface Fluid Based on Lattice Boltzmann Method," Computer-Aided Design and Computer Graphics. 23(1), 104110 (2011).

[12] J. Ojeda, A.Susín, "Hybrid Particle Lattice Boltzmann Shallow Water for Interactive Fluid Simulations”,GRAPP. IVAPP. 217-226, 2013

[13] R. P'eteri, S. Fazekas, M. J. Huiskes, ”Dyntex:a comprehensive database of dynamic textures" , Pattern Recognition Letters,Vol. 31, No. 12, pp. 1627-1632, 2010. 